\title{
Impacto del cambio de cobertura vegetal y del clima en la erosión del Nevado de Toluca
} Change of vegetable coverage impact in the erosion of the Nevado de Toluca

Homero Alonso ${ }^{1}$

Lamine Diakite ${ }^{2}$

Jaquelinne Rufino 3

${ }^{1}$ Facultad de Estudios Superiores Cuautitlán, Universidad Nacional Autónoma de México, Cuautitlán, Estado de México, México, alonso_m77@hotmail.com

Universidad Autónoma Chapingo, Texcoco, Estado de México, México, ceprae@gmail.com

${ }^{3}$ Instituto Tecnológico Superior de Huichapan, Huichapan, Hidalgo, México, jaquelinnera@hotmail.com

Autor para correspondencia: Homero Alonso, alonso_m77@hotmail.com

\section{Resumen}


El cambio climático implica la alteración espacial y temporal de variables climáticas, como temperatura y precipitación, las cuales, hidrológicamente, implican eventos extremos Iluvia-escurrimiento que producen pérdidas humanas, económicas y ecológicas en la parte baja de las cuencas; además, provocan la alteración del ciclo hidrológico. Por ello, se requiere en México del estudio de las cuencas como unidad fundamental, para identificarel efecto que el cambio de cobertura vegetal y el clima suscitan sobre sus características hidrológicas, a fin de proponer acciones de mitigación. En este estudio se evaluó el impacto del cambio de cobertura vegetal en la erosión hídrica mediante el uso de la técnica de álgebra de mapas, implementando un modelo hidrológico adaptado de la Ecuación Universal de Pérdida de Suelo (EUPS), y una serie multitemporal de imágenes multiespectrales Landsat y Spot de la década de 1980 y 2010. El trabajo se realizó en 52 microcuencas localizadas alrededor del volcán Nevado de Toluca. Los resultados mostraron que 36 de ellas presentaron incremento promedio en la tasa de erosión de $43.9 \%$, que se asocia con pérdida de la cobertura vegetal por incendios, labores antrópicas e incremento de la desertificación; las 16 restantes mostraron decremento debido a que tuvieron un cambio favorable en el uso del suelo y, por lo tanto, en su condición hidrológica; estos efectos se relacionan con los valores de las variables climáticas involucradas.

Palabras clave: percepción remota, modelo hidrológico, álgebra de mapas, cambio climático, uso de suelo, análisis multitemporal.

\section{Abstract}


The climatic change implies the spatial and temporal alteration of climatic variables such as temperature and precipitation which, in a hydrological way, imply extreme rain-draining events that produce human, economic and ecological losses in the lower part of the basins. It change the hydrological cycle to. For this reason, the study of basins is required in Mexico as a fundamental unit to identify the effect that climate change causes on its hydrological characteristics and to propose mitigation actions. This study evaluated the impact of climate change on water erosion by using the map algebra technique implementing a hydrological model adapted from the Universal Soil Loss Equation (EUPS) and a multitemporal series of multispectral images Landsat, and Spot of the decade of 1980 and 2010. The work was carried out in 52 subwathershed located around the Nevado de Toluca Volcano. The results showed that 36 sub-watersheds present an average increase in the erosion rate of $43.9 \%$, which is associated with the loss of vegetation cover due to fires, anthropogenic work and increased desertification. The remaining 16 showed decrement because they had favorable change in land use and therefore in their hydrological condition. These effects are related to the increase in the values of the climatic variables in recent years.

Keywords: Remote sensing, hydrological model, map algebra, climate change, land use, multitemporal analysis.

Recibido: 26/07/2017

Aceptado: 10/09/2019 
Tecnología y

Ciencias $\stackrel{\unlhd}{\unlhd}$ gua
2020, Instituto Mexicano de Tecnología del Agua

Open Access bajo la licencia CC BY-NC-SA 4.0

(https://creativecommons.org/licenses/by-nc-sa/4.0/)

\section{Introducción}

La temperatura de la Tierra se ha incrementado $0.74{ }^{\circ} \mathrm{C}$ durante los últimos cien años (IPCC, 2007) Los estudios regionales en el área de influencia del volcán Nevado de Toluca mostraron que la temperatura máxima se incrementó $1.87^{\circ} \mathrm{C}$ en los últimos 34 años (Diakite, 2008). Estos cambios inciden en el ciclo vegetativo de la cobertura que protege al suelo de la erosión, además las intensas sequías y elevadas temperaturas favorecen la propagación de incendios que acaban con esa protección, y aceleran la desertificación y degradación del suelo. Asimismo, el aumento de temperatura se acompaña de cambios en el índice de precipitación que tienen un impacto directo en la tasa de escurrimiento y, por lo tanto, de la erosión.

El cambio climático implica aumento de temperatura. Muestra de ello es lo sucedido en 1998. Los registros indican que ha sido el más caluroso de los últimos mil años, seguido de 2001 (NCDC, 2002); además, 9 de los 10 años más calurosos desde 1860 han ocurrido durante la década de 1990 (WMO, 2001). El calentamiento global implica que el ciclo hidrológico sea más intenso, incluyendo mayor precipitación y eventos más intensos de lluvia, como lo han mostrado los modelos de cambio climático aplicados al análisis de la precipitación y su intensidad. 
La pérdida de suelo por erosión es un problema local, nacional y global, que trae consigo problemas de fertilidad en los terrenos agrícolas, acarreo de contaminantes, azolve de embalses y degradación de hábitat acuático (Brady \& Weil, 2000). El estudio del proceso de erosión es relativamente reciente en México, comparado con lo que se ha hecho en EUA en lo que se refiere a su evaluación regional, y a su distribución espacial y temporal, teniendo su inicio a partir de la década de 1970 (Maass \& García-Oliva, 1990).

La estimación de erosión por métodos indirectos implica el uso de modelos basados en características físicas de la cuenca (roca, suelo, cobertura vegetal y relieve), pues determinan la tasa y tipo de ocurrencia de los procesos hidrológicos. No obstante, debido al escenario actual de los desastres naturales, hoy en día es necesario estudiar los posibles impactos regionales del clima sobre la erosión, lo cual se evalúa por medio de modelos hidrológicos adecuados, y sobre la base de escenarios climáticos representativos de cambios en la temperatura y precipitación. Con el objetivo de precisar la sensibilidad de las características hidrológicas de la cuenca por la alteración que provocan los cambios de cobertura vegetal y el clima, en este trabajo se estimó la pérdida de suelo en subcuencas utilizando la Ecuación Universal de Pérdida de Suelo Modificada (EUPSM) y el método del Número de Curva del USDA (1986) con un enfoque espacial, haciendo uso de información de cobertura vegetal para dos escenarios (1989 y 2014), obteniendo esta información de la clasificación de imágenes de satélite; con ello se identifica el efecto del cambio de cobertura y clima en el índice de erosión de las subcuencas del área circundante del Volcán Nevado de Toluca. 


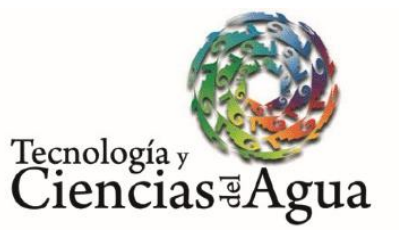

2020, Instituto Mexicano de Tecnología del Agua

Open Access bajo la licencia CC BY-NC-SA 4.0

(https://creativecommons.org/licenses/by-nc-sa/4.0/)

\section{Materiales y métodos}

La región de estudio se encuentra en el alto río Lerma y el alto río Balsas, en el radio de influencia de 60 kilómetros alrededor del volcán Nevado de Toluca, el cual se ubica en las coordenadas geográficas latitud $19^{\circ} 10^{\prime}$ y longitud $99^{\circ} 45^{\prime}$. El volcán está a $22 \mathrm{~km}$ al suroeste de la ciudad de Toluca, Estado de México; se le conoce también como Cinantécatl, y su formación es de traquita roja y andesita; al oriente, el volcán se liga con la Sierra de Tenango, los cerros de Jalatlaco y la Sierra del Ajusco.

Para desarrollar el trabajo se adquirieron 25 cartas topográficas escala 1:50 000 en formato vectorial y cuatro cartas edafológicas 1:250 000, además de los perfiles de suelo del Instituto Nacional de Estadística y Geografía (INEGI, 2015). También se utilizaron imágenes de satélite de los años 1989 y 2014; para 1989, dos imágenes Landsat ETM; para 2014, seis imágenes en formato pancromático y multiespectral de alta resolución SPOT $(\mathrm{V})$.

En cuanto a los trabajos de análisis espacial, se utilizó software especializado del ESRI (Environmental Systems Research Institute), como ArcGIS 10.3 (Iicencia ArcINFO), Erdas Imagine 9.1 y Envi/DL 4.5, además de otros especializados para análisis hidrológicos, como ArcHidro y River Tools 3.0. 
Se usó una estación de trabajo Dell Intel Core i2 a $2.12 \mathrm{GHz}$ y $8 \mathrm{~Gb}$ de memoria RAM para correr procesos de baja demanda de memoria. En los procesos de alta demanda, se utilizó una estación Dell Intel con dos procesadores Core i7 a $4.12 \mathrm{GHz}, 40 \mathrm{~Gb}$ de memoria RAM y dos discos duros de $2 \mathrm{~Tb}$.

\section{Métodos}

Para el desarrollo de este trabajo, se reunió la información correspondiente a cartas topográficas y edafológicas, a partir de las cuales se generó la información, con el propósito de alimentar el modelo de predicción de pérdida de suelo.

Con 25 cartas topográficas vectoriales con curvas de nivel a 10 metros de equidistancia, y con el software Envi 4.5, se generó el Modelo Digital de Elevación (MDE) de la zona de estudio, con resolución espacial de $1 \mathrm{~m}$. El procedimiento para dividir la zona en subcuencas consistió en el uso de la herramienta ArcHydro de ArcGIS 10.2.

La Ecuación Universal de Pérdida de Suelo Modificada (EUPSM) se desarrolló con el fin de estimar empíricamente la pérdida de suelo para cuencas pequeñas, al cambiar los factores de erosividad la eficiencia de transporte de sedimentos producidos por la energía cinética de la lluvia de la Ecuación Universal de Pérdida de Suelo (EUPS) (Wischmeier \& 
Smith, 1978) por una función del producto de volumen de escurrimiento de una tormenta por el escurrimiento pico (Williams, 1975). Esta ecuación se aplica para pérdida de suelo anual promedio o para estimar la pérdida diaria; en este estudio se estimó la pérdida anual. La ecuación de la EUPSM es como sigue:

$y=11.8\left(Q * q_{p}\right)^{0.56} K C S L P$

Donde:

$y=$ pérdida de suelo, en (t/ha).

$Q=$ escurrimiento, en $\left(\mathrm{m}^{3}\right)$.

$q_{p}=$ escurrimiento pico, en $\left(\mathrm{m}^{3} / \mathrm{s}\right)$.

$K, C, S L$ y $P=$ factores estándar de erosividad del suelo $(K) ;$ manejo de cultivos (cobertura vegetal $C$ ); grado de la pendiente $(S)$; longitud de pendiente $(L)$, y prácticas mecánicas de conservación para el control de la erosión $(P)$ de la ecuación EUPS.

El procedimiento para la aplicación del modelo de la EUPSM en cada una de las subcuencas es como se indica en el diagrama de la Figura 1, y el procedimiento para obtener los mapas de cada uno de los factores de la ecuación es como se describe enseguida. 


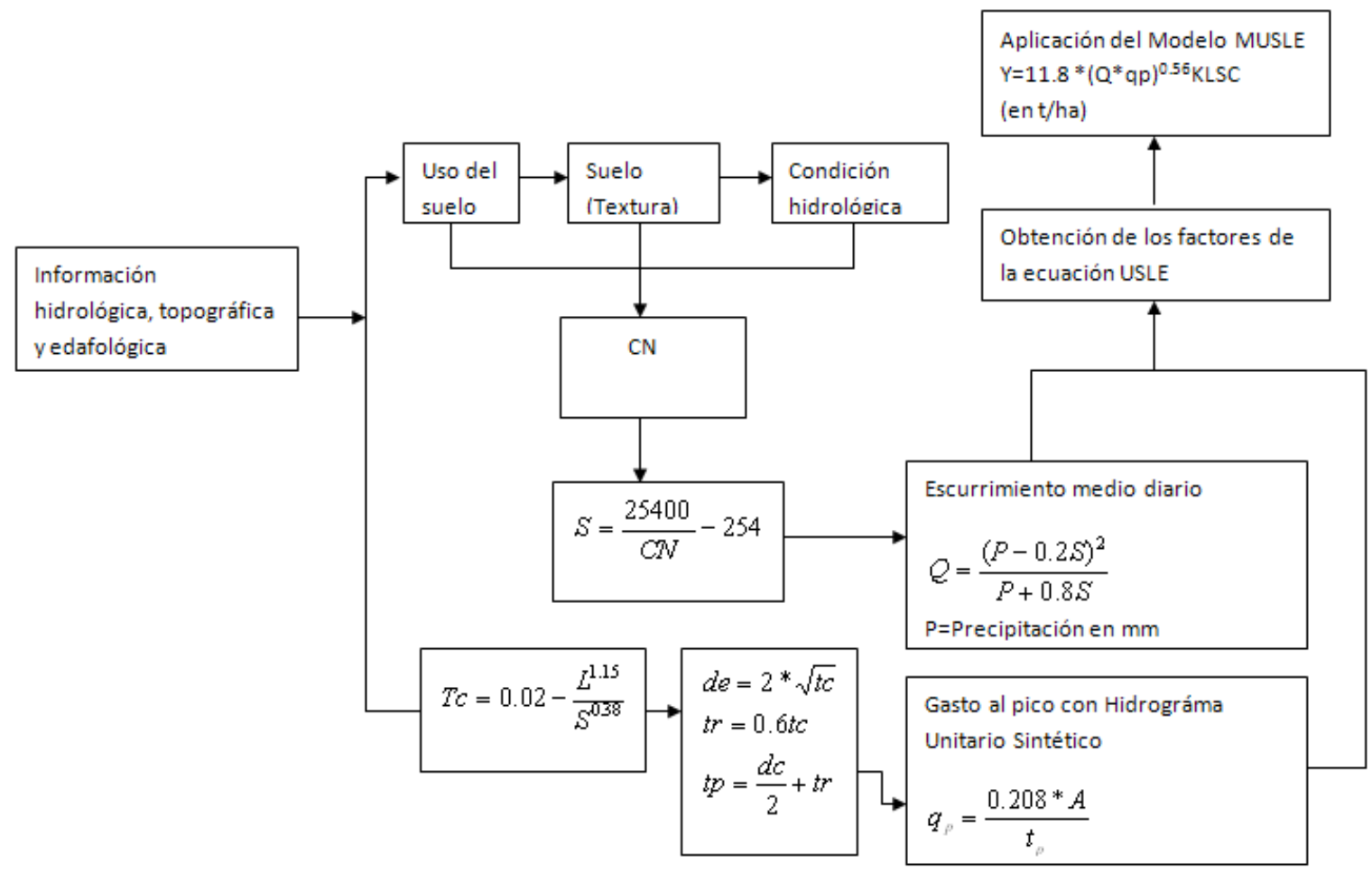

Figura 1. Metodología para la estimación de pérdida de suelo con la ecuación EUPSM.

El procedimiento para evaluar el factor $K$ con el método de la Organización de las Naciones Unidas para la Alimentación y la Agricultura (FAO, por sus siglas en inglés) consistió en determinar las unidades de suelo dentro de la zona de estudio y su textura de acuerdo con la clasificación de la Comisión de Estudios del Territorio Nacional (Cetenal); luego, con los datos de orden de suelo y textura, se utilizó la tabla de la FAO para obtener $K$.

Para obtener el factor $L$ se utilizó la Ecuación (2), recomendada por la metodología del manual de predicción de pérdida de suelos (Figueroa et al., 1991): 
2020, Instituto Mexicano de Tecnología del Agua

$L=(\lambda / 22.1)^{m}$

Donde:

$\Lambda=$ longitud de la pendiente, en $\mathrm{m} y$.

$m$ = exponente influenciado por la interacción de la longitud de la pendiente con la inclinación, así como las propiedades del suelo, tipo de vegetación y prácticas de conservación; adimensional.

Para obtener el factor $S$, que involucra la pendiente, se parte del cálculo de $S_{t}$ con la Ecuación (3):

$S_{t}=\frac{D_{n}}{\lambda} * 100$

Donde:

$S_{t}=$ pendiente del terreno, en $\%$.

$D_{n}=$ desnivel en metros, en $\mathrm{m} / \mathrm{m} y$.

$\Lambda=$ longitud de la pendiente, en $\mathrm{m}$.

El factor $S$ está en función de $S t$ y se calcula utilizando la Ecuación (4):

$S=0.065+0.045 S_{t}+0.0065 S_{t}^{2}$ 
Por lo general, el factor $C$ se da en términos del valor promedio anual para una combinación de sistemas de cultivo, manejo y lluvias. En este caso, las pérdidas relativas de suelo debidas al factor $C$ se obtuvieron de las tablas de valores del factor $C$, según las recomendaciones de Wischmeiery Smith (1978).

Para calcular el escurrimiento, se empleó el método del Número de Curva, desarrollado por el Servicio de Conservación de Suelos de EUA (USSCS, por sus siglas en inglés).

En este método, a cada tipo de suelo se le asigna un grupo hidrológico, que se basan en la capacidad de infiltración; ello se relaciona con la conductividad hidráulica de la superficie del suelo saturado.

A partir de las cartas edafológicas 1:250 000 y de la información de los perfiles de suelo, se generó el mapa de grupo hidrológico que está en función del tipo de suelo, textura y otras propiedades físicas del mismo.

El mapa de uso de suelo se obtuvo de la clasificación supervisada de las imágenes de satélite, para lo que se utilizó el algoritmo de máxima verosimilitud como regla de decisión para la clasificación de las imágenes en los dos periodos.

Este algoritmo tiene su base en asumir que las muestras de cada clase y cada banda se distribuyen de forma normal, así que la regla de decisión para un píxel $X$ desconocido es asignar $X$ a la clase $c$ sólo si se cumple con la probabilidad:

$$
p_{c} \geq p_{i}
$$


Donde $i=1,2,3, \ldots, m$ posibles clases, y $p_{c}$ es el píxel medio de clase y se calcula con la Ecuación (5) como:

$p_{c}=\left\{-0.5 \log _{e}\left[\operatorname{det}\left(V_{c}\right)\right]\right\}-\left[0.5\left(X-M_{c}\right)^{T} V_{c}^{-1}\left(X-M_{c}\right)\right]$

Donde:

$M_{c}=$ vector medio de clase.

$V_{c}=$ matriz de covarianza

$\operatorname{det}\left(V_{c}\right)=$ determinante de la matriz de covarianza $V_{c}$.

Así, para asignar el vector $X$ de un píxel desconocido dentro de una clase, la regla de decisión primero calcula el píxel medio de clase, luego se asigna a la que tiene el valor más grande de probabilidad (ITT, 2008).

El escurrimiento estimado por el método de Número de Curva (NC) se obtuvo con la Ecuación (6):

$Q=\frac{(P-0.2 * S)^{2}}{(P+0.8 * S)}$

Donde:

$S=(25400 / N C)-254$, adimensional.

$P=$ precipitación, en $\mathrm{mm}$.

$N C=$ número de curva de escurrimiento, adimensional $y$.

$Q=$ escurrimiento, en $\mathrm{mm}$. 
Para usar esta ecuación se generó un mapa de precipitación anual de cada periodo con información del Extractor Rápido de Información Climatológica ERIC III (IMTA, 2013). Con la información de Grupo Hidrológico y de Uso de Suelo derivado de la clasificación de imágenes se generó el mapa del NC, y con este último el de $S$. El valor de $Q$ se transformó a $\mathrm{m}^{3}$, para así obtener el volumen escurrido. Para calcular el gasto al pico en cada subcuenca se utilizó la Ecuación (7):

$q_{p}=\frac{0.208 \mathrm{~A}}{t_{p}}$

Donde:

$q_{p}=$ es el gasto al pico, en $\mathrm{m}^{3} / \mathrm{s} / \mathrm{mm}$.

$t_{p}=$ es el tiempo al pico, en $\mathrm{h} y$.

$A=$ es el área de la cuenca, en $\mathrm{km}^{2}$.

Por tanto, al calcular los parámetros de la Ecuación (7) y al resolverla en cada subcuenca, se generó el mapa ráster de gasto al pico $q_{p}$.

Con los parámetros de la Ecuación (1), representados a través de mapas tipo ráster, se implementó el modelo EUPSM en la herramienta Raster Calculator de ArcMap 10.3. Esta herramienta integra la técnica del álgebra de mapas, por lo que con ella se generó el mapa de la erosión en los periodos de estudio. 
El modelo original de la EUPSM es $y=11.8\left(Q * q_{p}\right)^{0.56} \mathrm{KCSL}$ y la forma en que se implementó en el módulo de álgebra de mapas fue como $Y p=$ $11.8 *$ Pow $\left(\left(\left[Q_{-} m 3 \_r a s\right] *\left[q p_{-} \_m 3 \_r a s\right]\right), 0.56\right) *\left[K_{-} r a s\right] *\left[C_{-} r a s\right] . *\left[S L_{-} r a s\right]$.

\section{Resultados}

\section{Modelo digital de elevación}

El Modelo Digital de Elevación (MDE) resultante tiene la precisión espacial de un metro y fue generado en el software Envi 4.5. La precisión del MDE es importante, porque de éste se generan las líneas de parteaguas para definir las subcuencas hidrológicas. Las elevaciones máximas de 4680 msnmm se encuentran aproximadamente al centro del MDE, que corresponden a las del cráter del volcán Nevado de Toluca y los límites al noroeste, correspondiente a la Sierra de las Cruces; mientras que las elevaciones más bajas están hacia el sureste (estado de Guerrero) y suroeste (estado de Morelos). 


\section{Subcuencas hidrológicas}

Usando como insumo el $\mathrm{MDE}$, se generaron las subcuencas dentro de un radio de $60 \mathrm{~km}$ del área de influencia del Nevado de Toluca. De este proceso resultaron 52 de ellas según se muestran en la Figura 2, las cuales se caracterizaron geométricamente según las define el relieve de la región que corresponde a montañas con disección, cordilleras y serrezuelas recientes con escudos. En las subcuencas, la superficie promedio es de $183 \mathrm{~km}^{2}$, la pendiente media es de $12 \%$, la red de drenaje es de tercer orden y la forma predominante es alargada. 
Tecnología y

2020, Instituto Mexicano de Tecnología del Agua

Ciencias $₫$ Agua

Open Access bajola licencia CC BY-NC-SA 4.0

(https://creativecommons.org/licenses/by-nc-sa/4.0/)

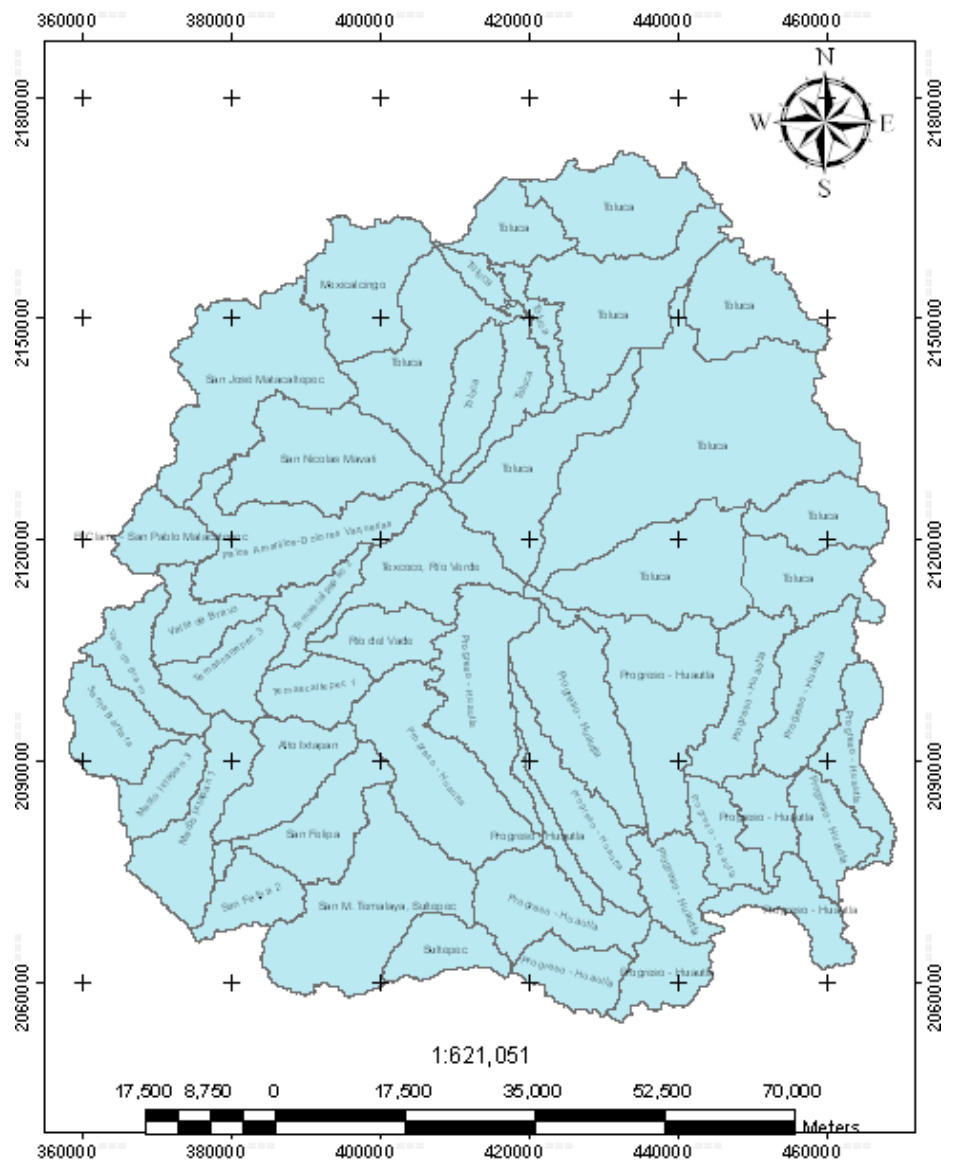

Figura 2. Subcuencas en el radio de $60 \mathrm{~km}$ respecto al Nevado de Toluca

\section{Factores de la ecuación EUPSM}


Los mapas resultantes de los factores $K, L S, C$ tienen resolución espacial de 10 metros que, para el álgebra de mapas, corresponde al tamaño de píxel de la imagen de satélite.

El mapa del factor $K$ muestra valores más altos (0.079) en las subcuencas ubicadas al suroeste del Nevado de Toluca, que corresponden con los valores máximos de pendiente; mientras que en las subcuencas que descienden de él hacia el Valle de Toluca, se tienen los valores mínimos.

El factor $L S$ por inclinación y longitud de pendiente es de 0.995 en las zonas donde la topografía es accidentada y corresponde a las subcuencas que descienden del Volcán y hacia la zona del sur, en las que la superficie se caracteriza por su relieve accidentado; mientras que en el Valle de Toluca se tienen valores de 0.34 que corresponden a las subcuencas hacia el norte.

El factor $C$ debido a la cobertura vegetal y manejo del suelo está relacionado con el mapa de uso de suelo. El mapa del factor $C$ tiene valores que van entre 0 y 1 , teniendo valores más bajos en los lugares donde existen bosques con buena cobertura, como en las subcuencas; Río del Valle, Toluca, Río Verde y Progreso, que descienden del Nevado de Toluca y de la Sierra de las cruces (noreste del área de estudio); por su parte, en el Valle y hacia Temazcalcingo, los valores de $C$ son cercanos o iguales a 1 debido a la escasa cobertura vegetal, donde se localizan las subcuencas de Progreso-Huautla, Temascaltepec, Valle de Bravo, San Felipe y Medio Ixtapan. 
Teçnología y

Ciencias $\stackrel{\unlhd}{\unlhd}$ gua
2020, Instituto Mexicano de Tecnología del Agua

Open Access bajo la licencia CC BY-NC-SA 4.0

(https://creativecommons.org/licenses/by-nc-sa/4.0/)

\section{Uso de suelo y grupo hidrológico}

La Figura 3 muestra la distribución de los grupos hidrológicos de suelo, donde se identificaron cuatro grupos que varían de acuerdo con las características físicas del suelo. El grupo dominante es el B, con 66.9\% del área, que corresponde a suelo de textura media con moderada permeabilidad en estado mojado. Le sigue el grupo D, con $14.7 \%$, correspondiente a suelos muy impermeables que favorecen el escurrimiento y por lo tanto la erosión. 
Tecnología y

Ciencias $₫$ Agua
2020, Instituto Mexicano de Tecnología del Agua

Open Access bajola licencia CC BY-NC-SA 4.0

(https://creativecommons.org/licenses/by-nc-sa/4.0/)

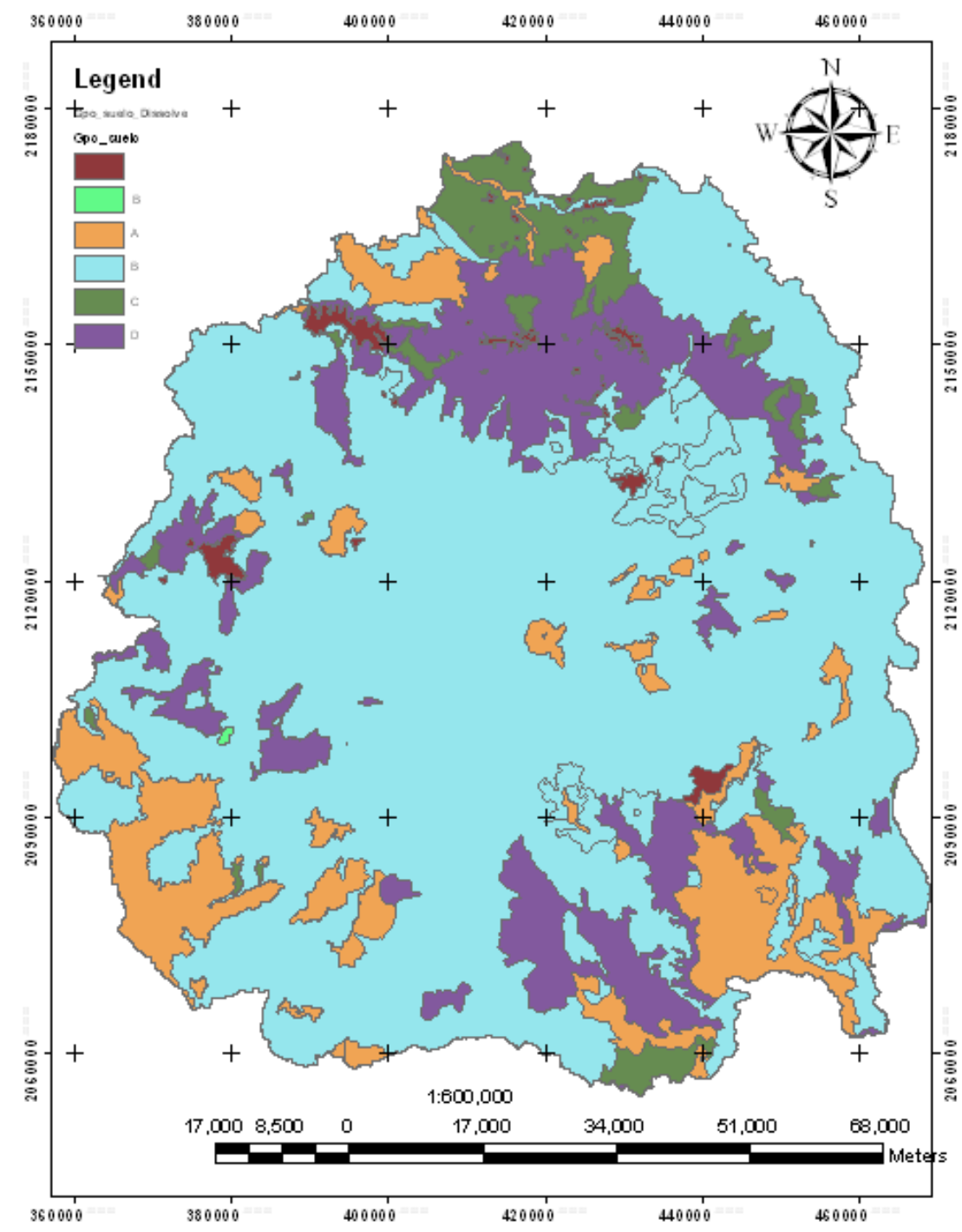

Figura 3. Mapa de grupos hidrológicos de suelo.

El resultado de la clasificación digital de imágenes de satélite muestra seis clases de uso de suelo, que se observan en la Figura 4 para los dos periodos analizados, que corresponden a: 1) cuerpos de agua; 2) bosque; 3) matorral; 4) suelo desnudo; 5) agricultura, y 6) zonas urbanas. Las clases fueron definidas de acuerdo con la nomenclatura de uso de suelo del Instituto Nacional de Estadística y Geografía. 
Tecnología y

Ciencias $₫$ Agua
2020, Instituto Mexicano de Tecnología del Agua

Open Access bajo la licencia CC BY-NC-SA 4.0

(https://creativecommons.org/licenses/by-nc-sa/4.0/)
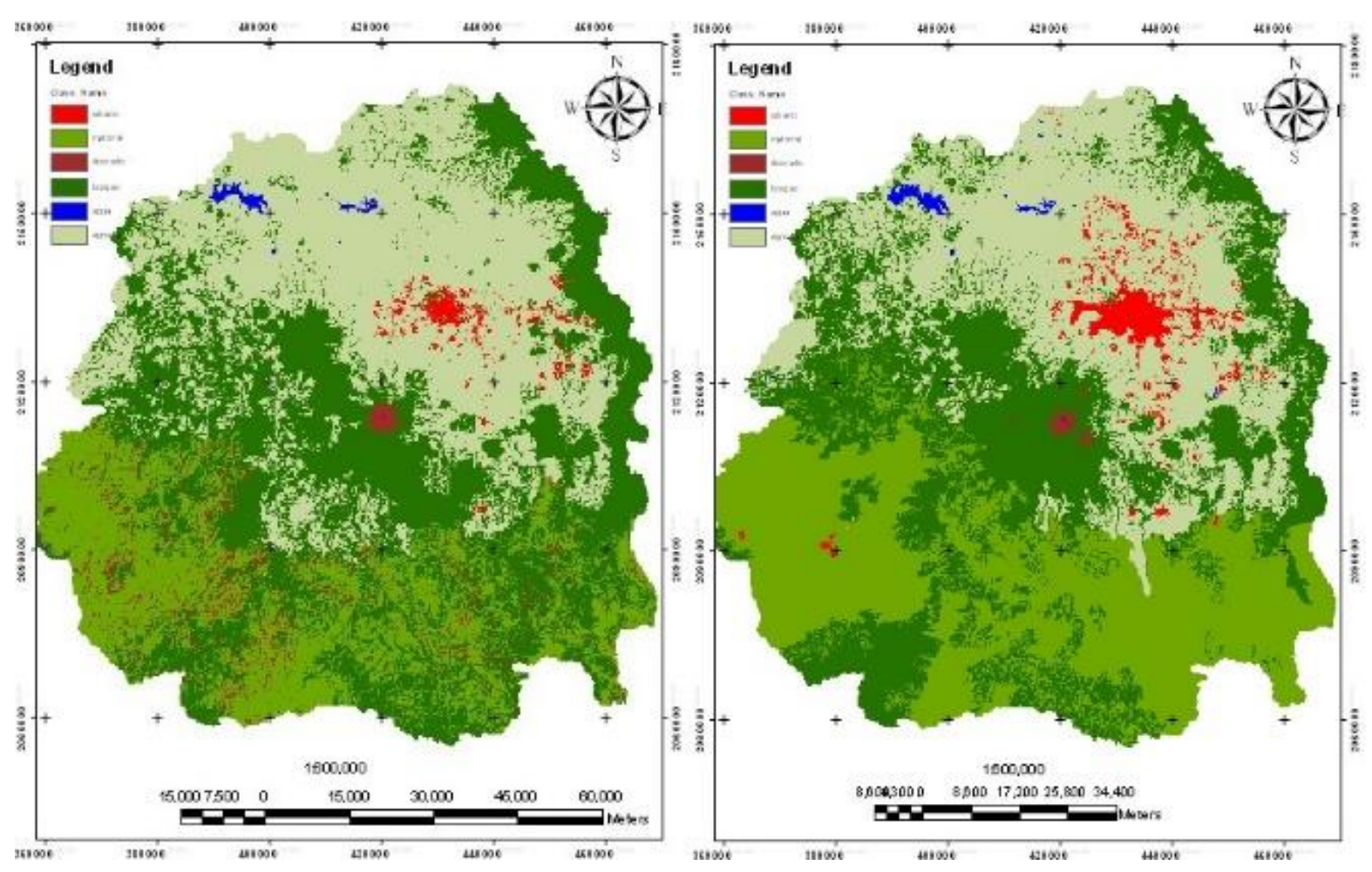

Figura 4. Uso de suelo en 1989 (izquierda); uso de suelo en 2014 (derecha).

La Figura 4 (derecha) muestra cómo, en la parte sur y sureste del área de estudio, se presentó cambio en el uso del suelo, el cual se debe a la deforestación y al sistema roza-tumba-quema (R-T-Q) para la actividad agrícola; también se asocia con los incendios causados por las altas temperaturas, ya que en el estudio de Diakite (2008), se encontró incremento significativo en la temperatura del área circundante al Nevado de Toluca, además coincide con los estudios donde el incremento significativo de la temperatura es el factor que detona los incendios, como lo indican González, Lara, Urrutia y Bosnich (2011); Yong (2003); 
Bonebrake et al. (2014); Guariguata (2009); Laucirica, Mancino, Uboldi y Michalijos (2011), y Huerta-Martínez e Ibarra-Montoya (2016).

\section{Escurrimiento}

Para la estimación del escurrimiento por el método del Servicio de Conservación de Suelos (SCS) se aplicó la Ecuación (6), para lo que se obtuvieron los mapas del Número de Curva ( $\mathrm{CN}$, por sus siglas en inglés), como se muestra en la Figura 5. Los valores más grandes de CN favorecen el escurrimiento por la condición hidrológica del suelo y su cobertura muy pobre. La variación en el valor de $C N$ de un periodo a otro depende básicamente del cambio en el uso de suelo, como la cobertura vegetal, pues las características físicas del suelo no se modificaron en el tiempo. 

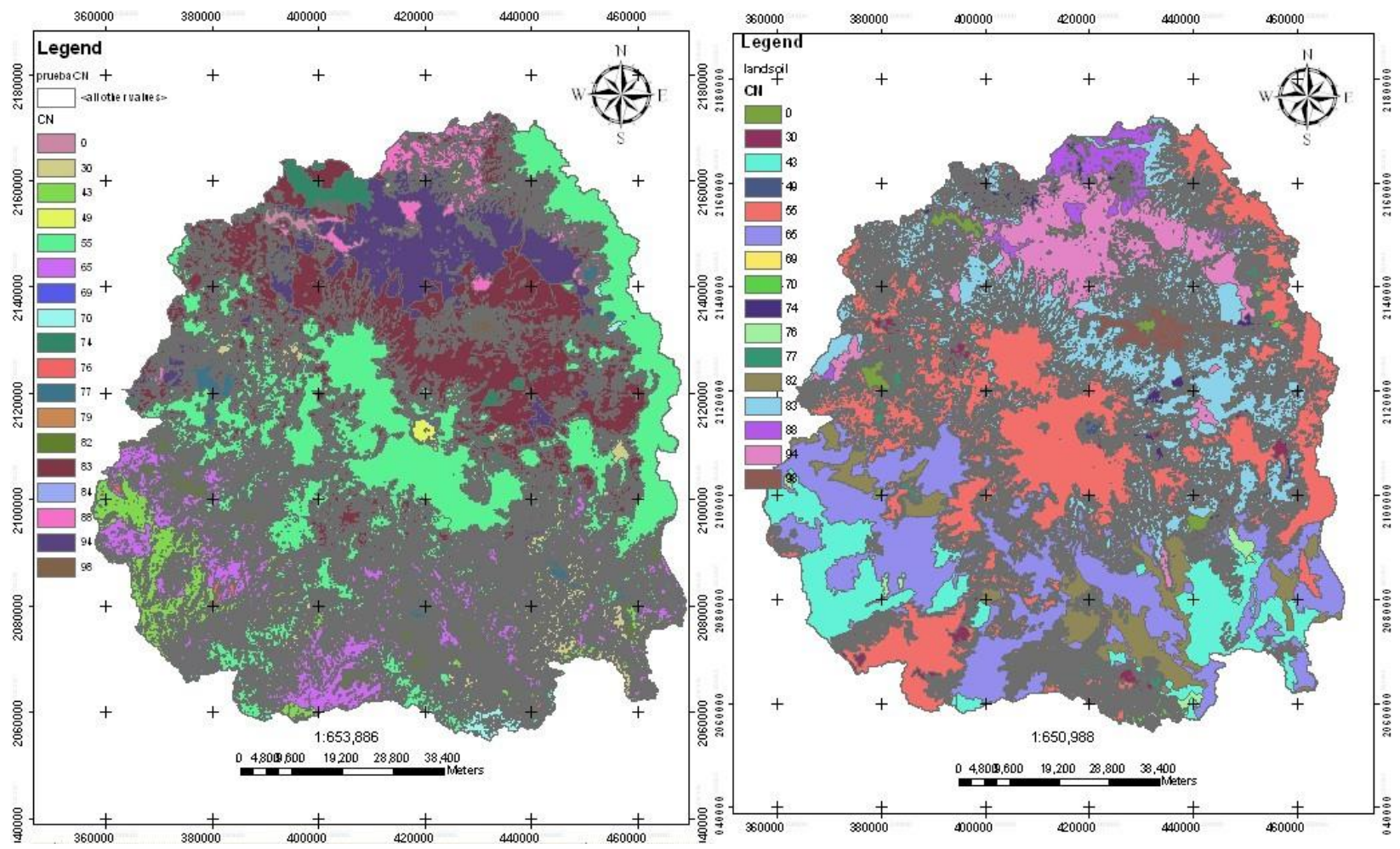

Figura 5. Número de curva $(C N)$ en 1989 (izquierda) y en 2014 (derecha).

El escurrimiento generado con el método del Número de Curva para cada periodo muestra variación espacial debido a los factores de grupo hidrológico y cobertura vegetal que intervienen en su cálculo; esta variabilidad va de 52 a $108 \mathrm{~m}^{3} /$ ha en 1989 y de 41 a $131.3 \mathrm{~m}^{3} / \mathrm{ha}$ en 2014. La variación de escurrimiento se refleja en la erosión; las áreas de menor escurrimiento se localizan en las subcuencas que descienden del Nevado de Toluca y de la Sierra de las Cruces, y las áreas de mayor escurrimiento se localizan en las subcuencas de Progreso-Huautla al sur del área de estudio. 
Tecnología y

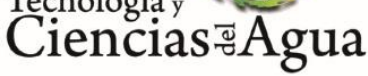

2020, Instituto Mexicano de Tecnología del Agua

Open Access bajo la licencia CC BY-NC-SA 4.0

(https://creativecommons.org/licenses/by-nc-sa/4.0/)

\section{Mapa de erosión}

Los mapas de erosión de cada periodo se muestran en la Figura 6 . En el mapa de 2014 se tienen valores más elevados de erosión en t/ha. De un total de 52 subcuencas en el área estudiada, 36 mostraron incremento positivo en la erosión respecto a 1989.
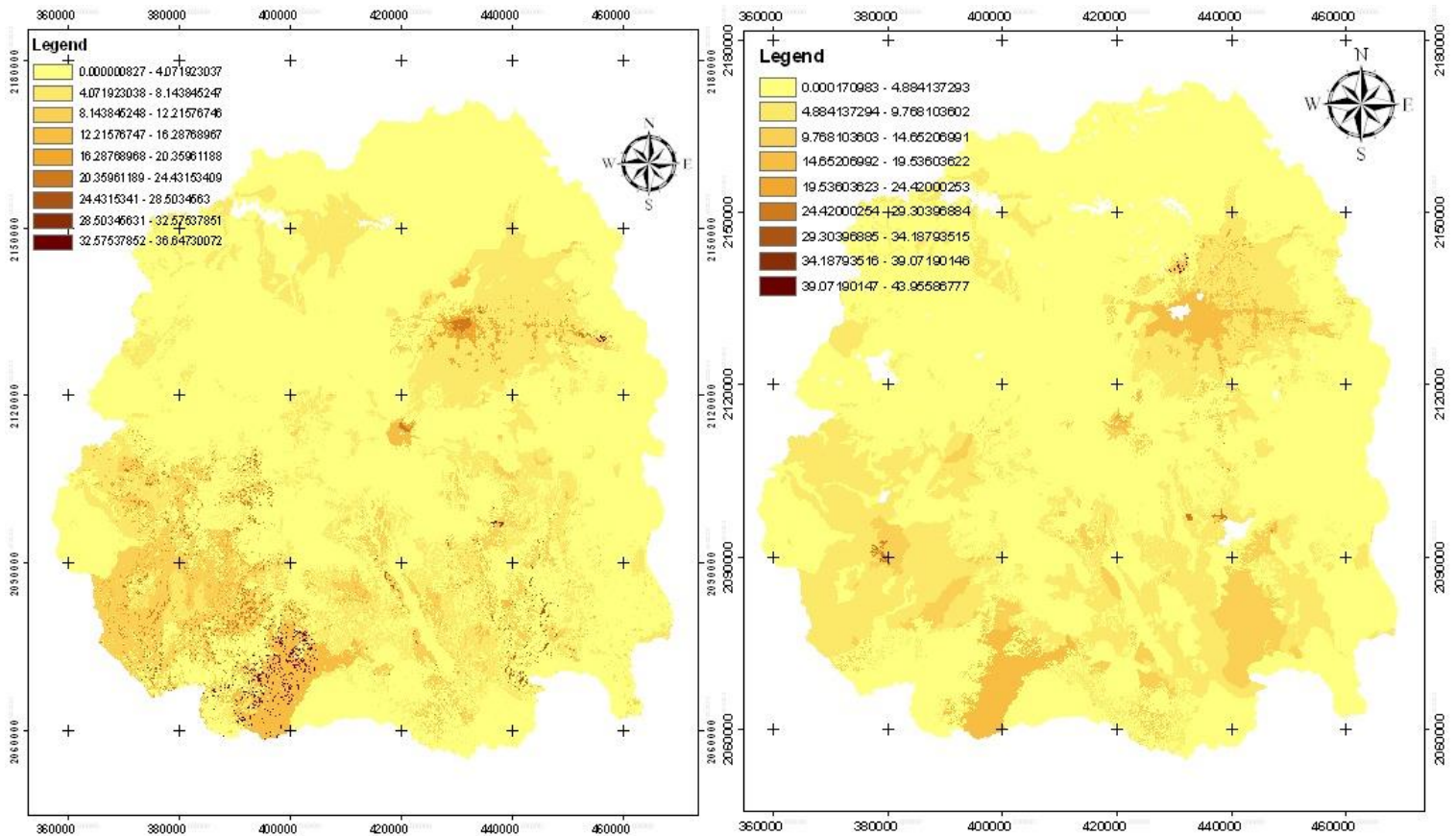

Figura 6. Erosión (t/ha/año) en 1989 (izquierda y 2014 (derecha).

Las subcuencas con valores negativos se deben a un cambio favorable en sus condiciones de cobertura vegetal, es decir, se 
regeneraron algunos bosques o pastizales que por algún tiempo fueron utilizados para la agricultura.

Con base en los niveles de erosión (FAO, 1980) y a la clasificación geodinámica, las subcuencas estudiadas son agrupadas en dos unidades:

1. Medio estable: agrupa a subcuencas con erosión leve (0-10 t/ha/año); la formación del suelo domina sobre la erosión.

2. Medio penestable: se caracteriza por erosión moderada (10-50 t/ha/año), pero la formación de suelo compensa esta pérdida de suelo.

El incremento en la erosión se presentó principalmente en las subcuencas del Valle, así como aquellas que descienden del cráter, donde se redujo el área de cobertura vegetal dejando el suelo desprotegido; en tanto que la reducción en la tasa de pérdida de suelo se identificó hacia la parte noroeste y suroeste del volcán.

De acuerdo con los valores de pérdida de suelo, se tiene erosión leve, pues se producen de 0 a 10 t/ha/año y se caracteriza por ser de tipo laminar con pocas canaletas; presenta hasta un $25 \%$ del horizonte " $A$ " erosionado, por lo que corresponde al medio geodinámico estable.

También se presenta la erosión moderada en las zonas donde se producen de 10 a 50 t/ha/año; es de tipo canaletas, presentando de un 25 a 50\% del espesor del horizonte " $A$ " erosionado, que corresponde al medio geodinámico penestable.

\section{Conclusiones}


Se estimó la erosión utilizando la Ecuación Universal de Pérdida de Suelo Modificada (EUPSM) y resultó que de las 52 cuencas de estudio, 36 presentaron incremento promedio de $43.9 \%$ en la erosión hídrica asociado con el cambio de cobertura vegetal.

Se utilizó el método del Número de Curva para calcular el escurrimiento superficial en los dos periodos y se encontró sensibilidad al uso de suelo; es claro, ya que el tipo y porcentaje de la cobertura vegetal modifica las abstracciones iniciales, variando el escurrimiento y con ello la pérdida de suelo por erosión.

El clima es un factor que influye en las características hidrológicas de las cuencas, sobre todo en la cobertura vegetal, pasando de condiciones de buena cobertura a suelo desprotegido y/o desnudo debido a la vulnerabilidad a los incendios por las altas temperaturas, lo que incide en la pérdida de suelo por erosión.

De las estimaciones de erosión en ambos periodos se encontró evidencia de que los cambios de uso de suelo, sobre todo la pérdida de cobertura vegetal por incendios, incrementaron esta variable en el último periodo analizado; con ello se infiere que los incendios causados por altas temperaturas y por labores antrópicas inciden en el proceso de pérdida de suelo por erosión hídrica en las cuencas hidrográficas.

El modelo de erosión del Servicio de Conservación de Suelos de Estados Unidos mostró sensibilidad al factor de uso del suelo debido a los 
Tecnología y

Ciencias $\stackrel{\unlhd}{\unlhd}$ gua
2020, Instituto Mexicano de Tecnología del Agua

Open Access bajo la licencia CC BY-NC-SA 4.0

(https://creativecommons.org/licenses/by-nc-sa/4.0/)

cambios de cobertura vegetal que se identificaron en los periodos estudiados.

\section{Referencias}

Bonebrake, T. C., Syphard, A. D., Franklin, J., Anderson, K. E., Akçakaya, H. R., Mizerek, T., \& Regan, H. M. (2014). Fire management, managed relocation, and land conservation options for Long-Lived obligate seeding plants under global changes in climate, urbanization, and fire regime. Conservation Biology, 28(4), 10571067, DOI: $10.1111 /$ cobi.12253

Brady, N. C., \& Weil, R. R. (2000). Elements of the nature and properties of soils. Upper Saddle River, USA: Prentice-Hall.

Diakite, D.L. (2008). Detección y análisis del cambio climático en México mediante la percepción remota multitemporal de los glaciares tropicales y la evaluación de su impacto potencial sobre los ecosistemas circundantes. Caso del Pico de Orizaba y Nevado de Toluca. Informe Técnico. México, DF, México: Universidad Autónoma Chapingo.

Figueroa, S. B., Amante, H. G., Cortés, J. P., Osuna, E. S., Rodríguez, J. M., \& Morales, J. (1991). Manual de predicción de pérdidas de suelo por erosión. Montecillo, México: Colegio de Postgraduados.

FAO, Food and Agriculture Organization. (1980). Metodología para la evaluación de la degradación de los suelos. Roma, Italia: Food and Agriculture Organization. 
González, M. E., Lara, A., Urrutia, R., \& Bosnich, J. (2011). Cambio climático y su impacto potencial en la ocurrencia de incendios forestales en la zona centro-sur de chile (330-420 S). Bosque, 32(3), 215-219. Recuperado de http://dx.doi.org/10.4067/S071792002011000300002

Guariguata, M. R. (2009). El manejo forestal en el contexto de la adaptación al cambio climático. Revista de Estudios Sociales, (32), 98-113.

Huerta-Martínez, F. M., \& Ibarra-Montoya, J. L. (2016). Cambio climático y predicción de incendios al 2050 en el Bosque La Primavera, Jalisco. Revista Mexicana de Ciencias Forestales, 7(37), 39-50.

IMTA, Instituto Mexicano de Tecnología del Agua. (2013). Extractor Rápido de Información Climatológica ERIC III. (CD ROM). Jiutepec, México: Instituto Mexicano de Tecnología del Agua.

INEGI, Instituto Nacional de Estadística y Geografía. (2015). INEGI. Ciudad de México. Recuperado de https://www.inegi.org.mx/mapas

IPCC, International Panel of Climatic Change. (2007). Climate change 2007: The physical science basis. Contribution of Working Group I to the Four Assessment Report of the Intergovernmental Panel on Climate Change. Solomon, S., Qin, D., Manning, M., Chen, Z., Marquis, M., Averyt, K. B., Tignor, M., \& Miller, H. L. (eds.). Cambridge, UK, New York, USA: Cambridge University Press.

ITT, Introduces Automates Tools for ENVI. (2008) Exploring ENVI, Region of Interest and Clasification Techniques (pp. 131-132). Boulder, USA: Exelis Visual Information Solutions. 
Laucirica, J, Mancino, M., Uboldi, J., \& Michalijos, P. (2011). Cambio climático, turismo y riesgo de incendios forestales, de pasturas y de interfase, en el sudoeste bonaerense, su análisis con geotecnologías. Revista Geográfica de América Central, 2(juliodiciembre), 1-15.

Maass, J. M., \& García-Oliva, F. (1990). La investigación sobre la erosión de suelos en México. Un análisis de la literatura existente. Ciencia 41(3), 209-228.

NCDC, National Climatic Data Center. (2002). Climate of 2001. Annual Review. Asheville, USA: National Oceanic and Atmospheric Administration.

USDA, United State Department of Agriculture. (1986). Urban hydrology for smallwatersheds. Recuperado de http://cset.sp. utoledo.edu/ nkissoff/pdf/CIVE-3520/Modifiedtr55.pdf

Williams, J. R. (1975). Sediment-yield prediction with Universal Equation using runoff energy factor. In: Present and Prospective Technology for Predicting Sediment Yield and Sources (pp. 244-252) (ARS-S40). Washington, USA: U.S. Department of Agriculture.

Wischmeier, W. H., \& Smith, D. D. (1978). Predicting rainfall erosion losses a guide to conservation planning ( $\mathrm{AH}-537)$. Washington, USA: U.S. Department of Agriculture.

WMO, World Meteorological Organization. (2001). WMO statement on the status of the global climate in 2001. Geneva, Switzerland: World Meteorological Organization. 
2020, Instituto Mexicano de Tecnología del Agua

Tecnología y

Open Access bajo la licencia CC BY-NC-SA 4.0

(https://creativecommons.org/licenses/by-nc-sa/4.0/)

Yong, G. (2003). El cambio climático y los incendios: la naturaleza parece equilibrar la devastación ecológica hecha en los países subdesarrollados, consumiendo recursos forestales de "naciones ecológicamente educadas" como Estados Unidos, Canadá y las potencias europeas. Siempre, 50(2620), 56. 